\title{
THE IMPACT OF THE ALEXANDERWERK WP120 MILL UPPER MESH SIZE ON THE GRANULE PROPERTIES OF A LACTOSE/MICROCRYSTALLINE CELLULOSE PLACEBO FORMULATION
}

\author{
Karim Hassan $^{1}$, Marwan EIBagoury ${ }^{2}$ and Maryam Kotb ${ }^{3}$ \\ ${ }^{1}$ School of Pharmacy and Pharmaceutical Sciences, The University of Manchester, UK \\ ${ }^{2}$ Universität Ulm - Medizinische Fakultät, Ulm, Deutschland \\ ${ }^{3}$ University of The People, Pasadena, CA, USA \\ *E-mail: karimhassan66@outlook.com
}

\begin{abstract}
The continuous development of new active pharmaceutical ingredients (API) that are moisture sensitive, made roller compaction more popular, and thus encourages research into the different processing parameters whose modification may enhance the production of roller compactors. The Alexanderwerk WP120 is a roller compactor with an integrated two-stage mill that is used for small/medium scale batch manufacture. This study was carried out to determine the impact of upper mesh size of the integrated mill of the Alexanderwerk WP120 roller compactor on granule PSD, flow and compactability. The results showed that the upper mesh size may have a significant effect on PSD of lower solid fraction ribbon/wafer $(\leq 0.65 \mathrm{SF})$. However, these differences in PSD may not be sufficient to afford a significant change in bulk powder flow and compactability properties.

Keywords: Roller compaction, Alexanderwerk WP120, particle size distribution, upper mesh size
\end{abstract}

(C) RASĀYAN. All rights reserved

\section{INTRODUCTION}

Manufacture of tablets by dry granulation involves two methods; slugging and roller compaction. ${ }^{1}$ In roller compaction process, a flowing powder is continuously fed between two counter-rotating rolls, the first is fixed, while the second moves in one plane by the force of hydraulic pressure in order to form a compressed sheet. ${ }^{2,3}$ Many years ago, before the availability of continuous roller compactors, dry granulation method of choice was compressing briquette tablets in specific tablet presses and then breaking those tablets into granules. ${ }^{4}$ Roller compaction is a simple and economical process that can be easily scaled up. ${ }^{5}$ Most importantly, roller compaction can be applied with moisture/solvents and/or heat- sensitive active ingredients. $^{6-8}$ Therefore, nowadays the development of new active pharmaceutical ingredients (API) that are moisture sensitive, made roller compaction more popular. ${ }^{9}$ One drawback of roller compaction is that the process can alter the compactability of the final granule. ${ }^{10,11}$ Roller compactors are fitted with control devices that adjust compaction pressure, and speeds of rolling and powder feeding. These features provide high levels of process control and throughput, in addition to a formed compact of high quality. ${ }^{12}$ As is the case with the majority of pharmaceutical process machinery, a wide range of sizes is available and capable of operating with a wide range of throughputs and compaction forces, e.g. Alexanderwerk and Powtec. ${ }^{5}$

The Alexanderwerk WP120 is a roller compactor with an integrated two-stage mill that is used for small/medium scale batch manufacture. The two-stage mill consists of an upper coarse screen and a lower smaller fine screen. The granulator-screens are exchangeable, and the particle size of the final granule is determined by the mesh size and screen design. Alexanderwerk has recommended that the upper mesh size (UM) be equal to or greater than the ribbon thickness presented to the mill, with the rationale that effective milling would be achieved if the material is larger than the screen in all three dimensions. Upper mesh size may affect the particle size distribution (PSD) of the resulting granule, which may in turn affect granule flow, compactablity and tablet weight uniformity. ${ }^{13}$ 


\title{
RASĀYAN J. Chem.
}

\author{
Vol. 11 | No. 2 |460 - 464 | April - June | 2018
}

The study was performed using wafers manufactured using a Medel'Pharm Stylcam 100R rotary tablet press replicator; wafers can be manufactured with the same properties of ribbon, but with greater control and consistency with regard to solid fraction and thickness.

The aim of this study was to determine the impact of upper mesh size of the integrated mill of the Alexanderwerk WP120 roller compactor on granule PSD, flow and compactability. In order to achieve this aim, there were five objectives: manufacturing wafer batches of 0.65 and 0.75 solid fractions (SF) at thicknesses of 2.0, 2.7 and 3.5mm using a placebo formulation, measuring the tensile strength of the wafers, milling the wafers using a $2.5 \mathrm{~mm}$ or $3.15 \mathrm{~mm}$ upper mesh, with a $1 \mathrm{~mm}$ lower mesh, measuring the PSD and flow of the subsequent granule batches, and assessing the compactability of the granule batches.

\section{The used formulation}

\section{EXPERIMENTAL}

The lactose/microcrystalline cellulose placebo formulation used in this study had a true density of $1.55 \mathrm{~g} / \mathrm{cm}^{3}$ as determined using the Accupyc II 1340 helium pycnometer $(\mathrm{n}=3)$.

\section{Wafer Manufacture and Tensile Strength Measurements}

Wafers were manufactured using a Medel'Pharm Stylcam 100R rotary tablet press replicator fitted with 22 x $10 \mathrm{~mm}$ rectangular, flat faced tooling. Six batches were manufactured to represent two in-die solid fractions, 0.65 and 0.75 , at three in-die thicknesses of 2.0, 2.7 and $3.5 \mathrm{~mm}$. Tensile strength was calculated from the fracture force determined by a 3-point bend test using a Stable Micro Systems TA.XTplus Texture Analyser $(n=5)$. Wafer solid fraction was verified by envelope density using a GeoPyc 1360 .

\section{Milling and Particle Size Analysis}

Wafers were milled using an Alexanderwerk WP120 with two upper mesh sizes, 2.5 and $3.15 \mathrm{~mm}$. A $1 \mathrm{~mm}$ lower mesh and a rotor speed of 60rpm were used throughout. Particle size analysis was determined using a Sympatec GmbH QicPic image based particle sizing system using $\approx 1.5 \mathrm{~g}$ of riffled material $(\mathrm{n}=4)$.

\section{Placebo Blend and Granule Flow Properties}

Flow properties were determined using an Erwerka Granulate and Powder Flow Tester ( $\mathrm{n}=3$ ), and a Geldart Mark4 Angle of Repose tester $(\mathrm{n}=3)$. The former assessed the mean time for $\approx 100 \mathrm{~g}$ of material to flow through a $6 \mathrm{~mm}$ circular aperture, and the latter assessed the angle of the cone formed when $\approx 100 \mathrm{~g}$ of material was poured through a metal hopper onto an L-shaped plastic base unit.

\section{Granule Compactability Testing}

Extragranular excipients were added to each granule batch and the samples were compacted to a target solid fraction of 0.85 using a Medel'Pharm Stylcam 100R rotary tablet press replicator with 11.28 diameter, round, flat faced tooling. Six compacts were tested per granule batch; the weight, thickness and breaking force (using a tablet hardness tester) were measured, and the tensile strength was subsequently calculated.

\section{RESULTS AND DISCUSSION}

The PSD data (Fig.-1 and 2) revealed a bimodal distribution with a larger proportion of coarse, granular particles at $0.75 \mathrm{SF}$ versus $0.65 \mathrm{SF}$. Granule from the $3.5 \mathrm{~mm}$ wafers contained a significantly larger proportion of fine particles than those from the $2 \mathrm{~mm}$ wafers at both solid fractions $(\mathrm{P}<0.05)$. It has been proposed that the density distribution across a ribbon is not constant across the ribbon thickness ${ }^{9}$; therefore, thicker wafers may have a variable solid fraction throughout their thickness.

At $0.65 \mathrm{SF}$, the larger upper mesh size resulted in significantly fewer fine particles and more coarse particles by volume $(\mathrm{P}<0.05)$; however, this was not the case at $0.75 \mathrm{SF}$ (Fig.-3). Higher solid fraction resulted in significantly greater tensile strength $(\mathrm{P}<0.05)$ while wafer thickness had no significant effect on tensile strength (Fig.-4). Greater tensile strength wafers reduce to fines less readily thus explaining the larger volume of coarse particles observed at $0.75 \mathrm{SF}$ versus $0.65 \mathrm{SF}$. Improved flow rates were observed at the higher solid fraction and this was inversely proportional to wafer thickness. 
RASĀYAN J. Chem.

Vol. 11 | No. 2 | 460 - 464 | April - June | 2018

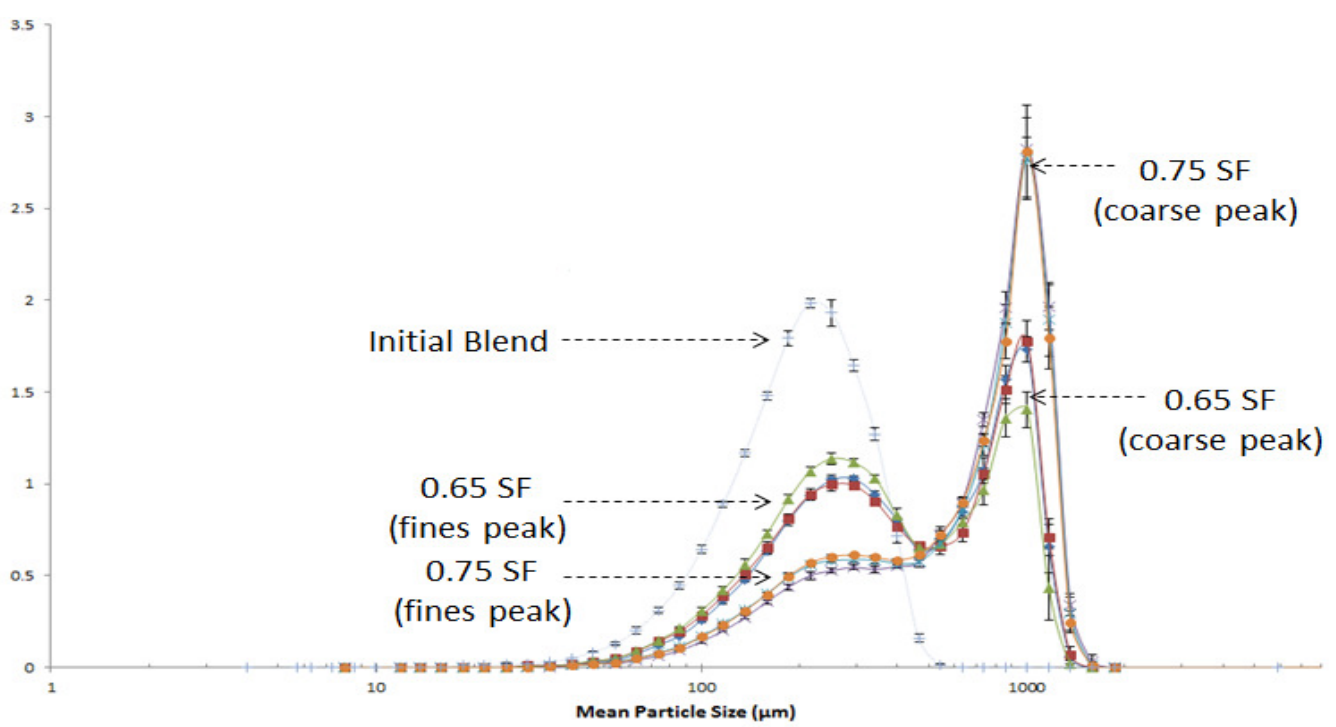

Fig.-1: Granule PSD Using 2.5mm Upper Mesh

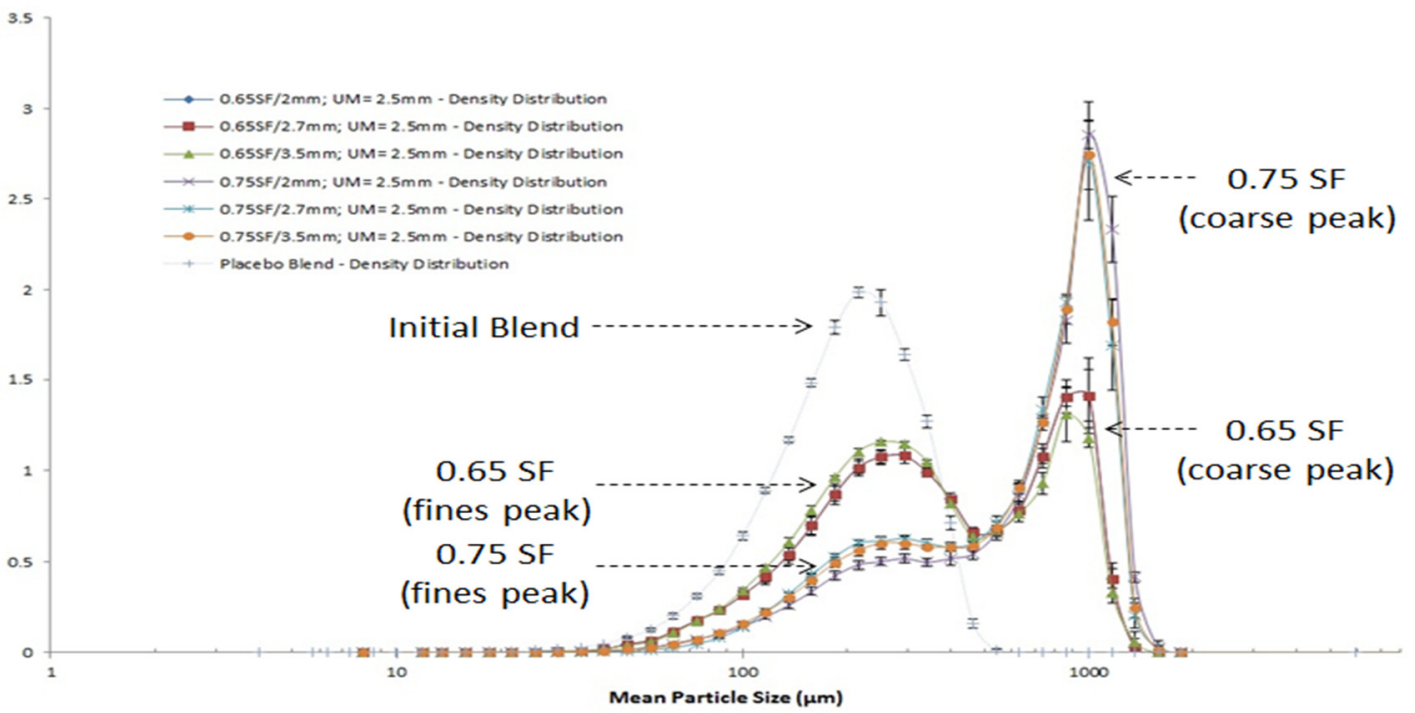

Fig.-2: Granule PSD Using 3.15mm Upper Mesh

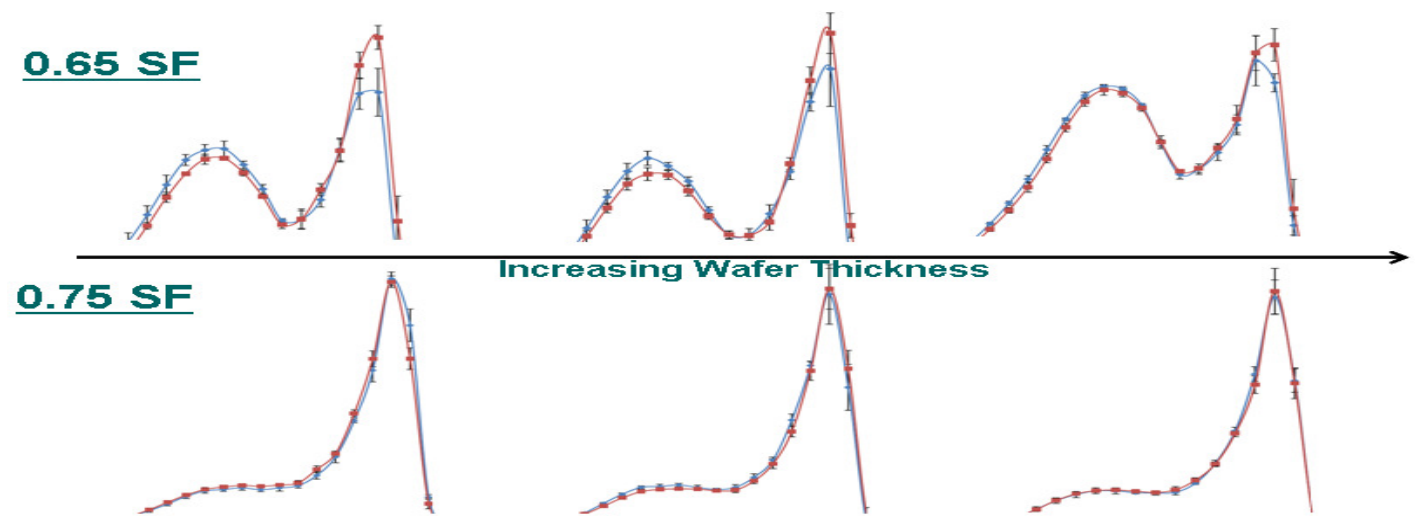

Fig.-3: Upper Mesh Comparison 
This is attributed to the increased coarse content at a higher solid fraction and the increased fines content with increasing wafer thickness; it is generally accepted that better flow is observed with granule comprising of larger particles versus the equivalent material containing a large proportion of fine particles. Upper mesh size had no significant effect on flow rates (results not shown). Wafer solid fraction had a significant correlation with granule compactability $(\mathrm{P}<0.05)$, with reduced compactability at higher solid fraction; while upper mesh size had no significant effect on granule compactability (Fig.-5). This is attributed to the greater work hardening effect at a higher solid fraction.

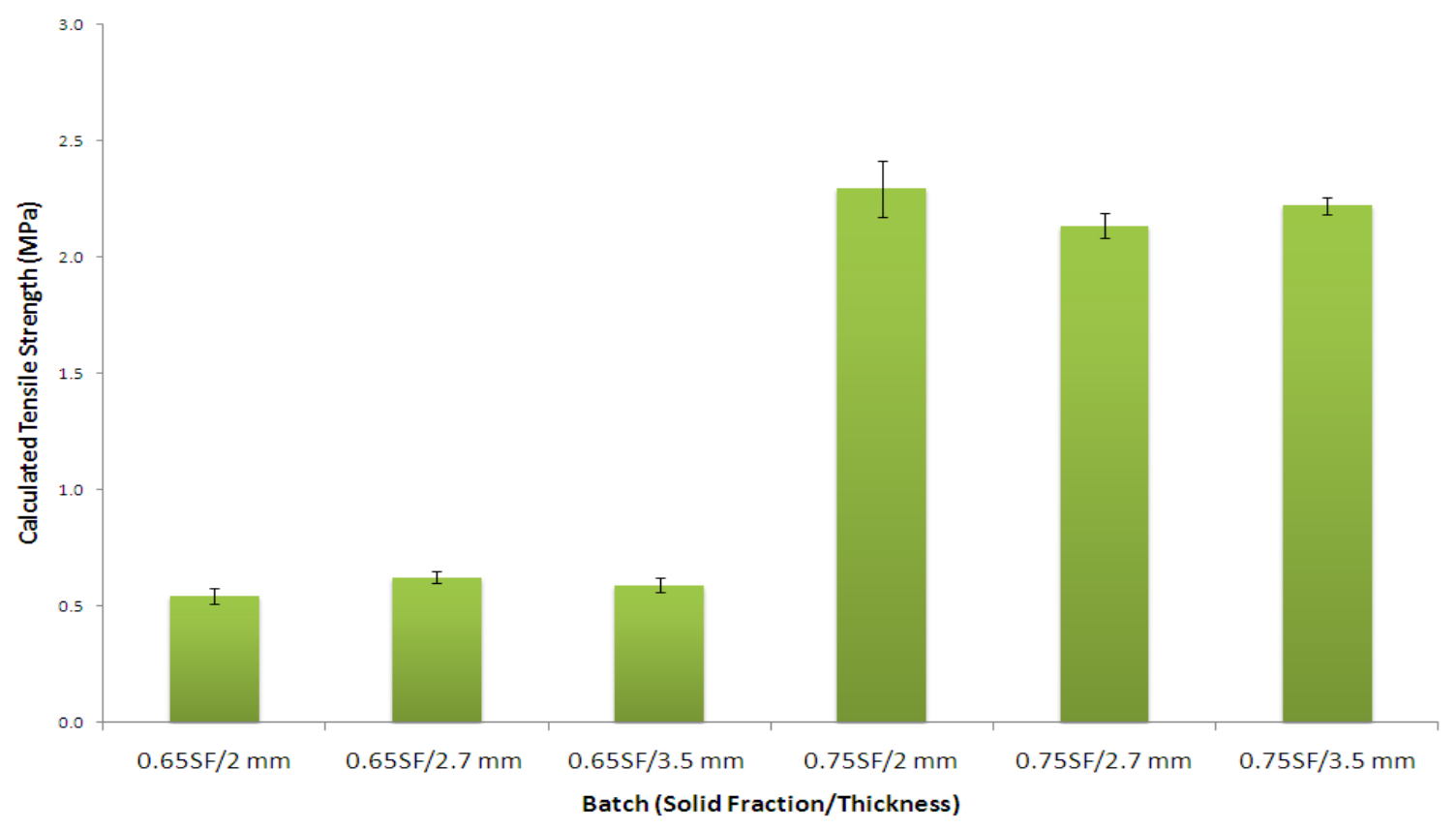

Fig.-4: Wafer Tensile Strength

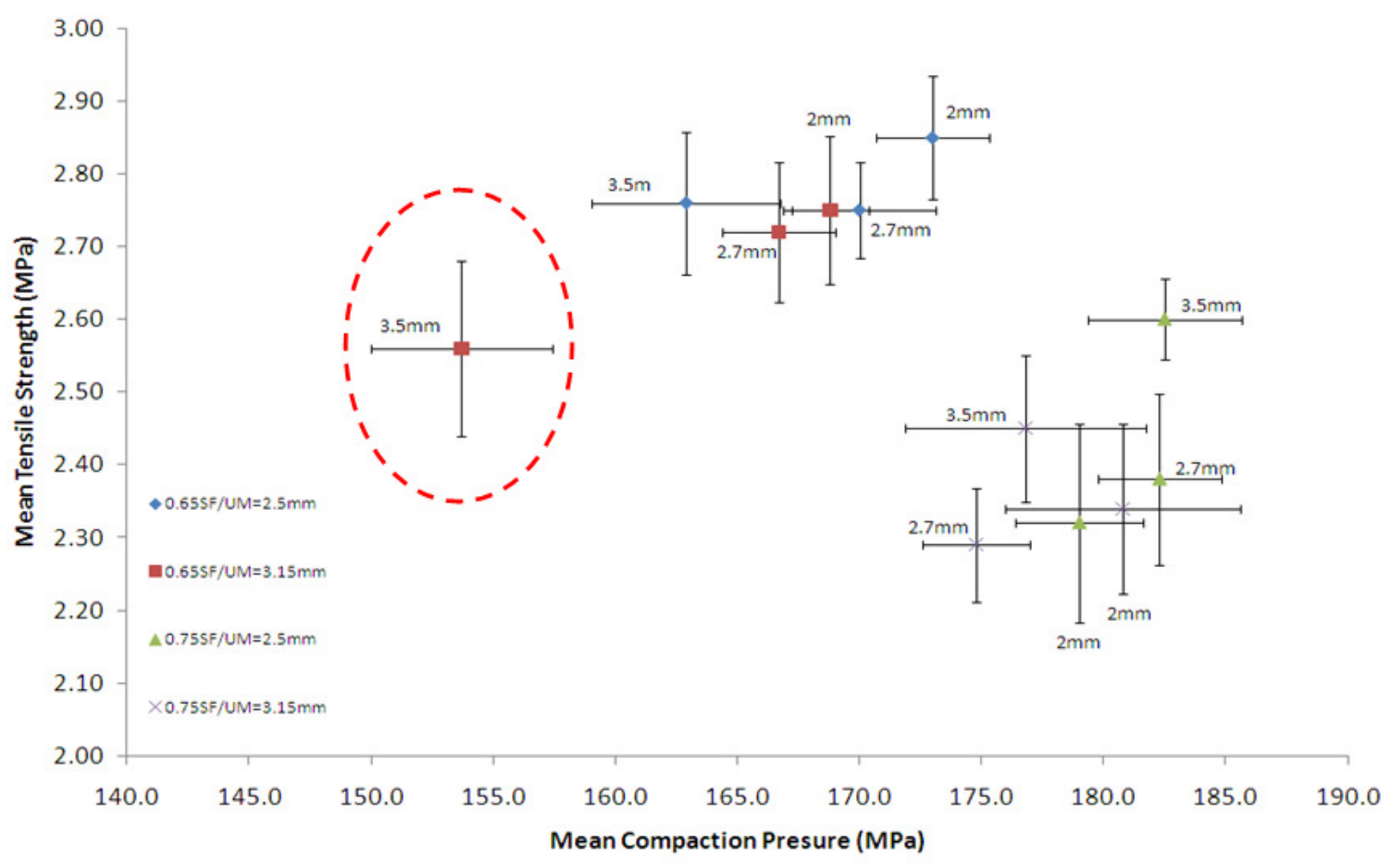

Fig.-5: Granule Compactability 


\section{CONCLUSION}

The upper mesh size may have a significant effect on PSD of lower solid fraction ribbon/wafer $(\leq 0.65 \mathrm{SF})$. However, these differences in PSD may not be sufficient to afford a significant change in bulk powder flow and compactability properties.

The wafer solid fraction/tensile strength has the most impact on PSD, flow and compactability; greater tensile strength wafers resulted in larger particles, improved flow and reduced compactability.

At the same solid fraction, increasing wafer thickness resulted in a significantly larger volume of fine particles; more prevalent at a lower solid fraction, and slower flow rate and improved compactability.

Overall, upper mesh size does not appear to be a critical process parameter.

\section{REFERENCES}

1. D. S. Jones, FASTtrack Pharmaceutics Dosage Form and Design, 2nd edition, Pharmaceutical Press, London, UK, p.280 (2016).

2. L. L. Augsburger and S. W. Hoag, Pharmaceutical Dosage Forms - Tablets, Third Edition, CRC Press, USA, p.309 (2016).

3. P. Pandey and R. Bharadwaj, Predictive Modeling of Pharmaceutical Unit Operations, Woodhead Publishing, UK, p.72 (2016).

4. A. T. Florence and J. Siepmann, Modern Pharmaceutics Volume 1: Basic Principles and Systems, Fifth Edition, CRC Press, USA, p.491 (2009).

5. M. E. Aulton and K. Taylor, Aulton's Pharmaceutics: The Design and Manufacture of Medicines, Elsevier Health Sciences, UK, p.485 (2013).

6. M. E. Aulton, Pharmaceutics: The Science of Dosage Form Design, Churchill Livingstone, UK (2002).

7. B. Bennett and G. Cole, Pharmaceutical Production: An Engineering Guide, IChemE, UK, p.112 (2003).

8. I. Jeon, M. Bikrom, G. Tiziana, V. Thierry and B. Gabriele, Pharmaceutical Technology Europe, 21, 12,2009

9. J. F. Gamble, M. Tobyn, A. B. Dennis and T. Shah, Pharmaceutical Development and Technology, 15, 3, 2010, DOI: 10.3109/10837450903095342

10. D. M. Parikh, Handbook of Pharmaceutical Granulation Technology, Third Edition, CRC press, USA (2016).

11. S. Malkowska and K. A. Khan, Drug Development and Industrial Pharmacy, 9, 3, 1983, DOI: 10.3109/03639048309044678

12. D. M. Parikh, Handbook of Pharmaceutical Granulation Technology, CRC Press, USA, p.103 (1997).

13. A. GmbH, Laboratory and Pilot Production Machine for The Pharmaceutical Industry WP 120 Pharma. Remscheid, Germany. p. 1-6.

[RJC-2059/2018] 